\title{
De la Sensibilisation des Populations à la Gestion de l'Environnement Urbain dans les Quartiers Précaires De la Ville d'Abidjan
}

\section{Traoré KASSOUM}

Unité de Formation et de Recherche des Sciences et Gestion de l'Environnement (Option politique environnementale)/ Université d'Abobo-Adjamé / Côte d'IVoire

\section{RESUME}

La prolifération des quartiers précaires dans la ville d'Abidjan qui résulte d'une urbanisation rapide et non maîtrisée est un réel problème pour les pouvoirs publics et pour l'ensemble de la société ivoirienne. Cette situation ne surprend guère dans la mesure où la ville d'Abidjan est apparue depuis les années 1970 comme une véritable capitale cosmopolite de la sous région. Elle résulte du développement de l'industrie, du commerce et des autres secteurs d'activité qui se concentrent dans la région d'Abidjan.

La crise économique que connaît le pays, et son corollaire qu'est la paupérisation des couches les plus vulnérables de la population urbaine, posent le problème de la gestion des quartiers précaires dont le nombre ne cesse de croître au fil des années. La densification des populations dans ces types de quartiers sans commodités provoque des problèmes de santé liés justement à la dégradation de l'environnement.

Les populations de ces quartiers- généralement démuniesont peu d'information sur la gestion de l'environnement urbain, de sorte que celles-ci s'intéressent peu à la gestion de leur cadre de vie. Il n'existe, à l'heure actuelle en Côte d'Ivoire, aucune action systématisée et coordonnée en matière d'information et de sensibilisation environnementale (SISSOKO Alain, 1993). La sensibilisation des populations est encore insuffisante. Le présent article tente de montrer les insuffisances dans ce domaine.

Mots clés : sensibilisation, gestion de l'environnement, quartiers précaires, Abidjan 


\section{INTRODUCTION}

Carrefour de plusieurs flux migratoires de la sous-région, la ville d'Abidjan, avec environ 4 millions d'habitants, constitue depuis plusieurs années un véritable pôle d'attraction. Notons aussi qu'à la suite du déclenchement du conflit armé du 19 septembre 2002, la ville a accueilli plus de $75 \%$ des déplacés internes $(1)$. Dans ce contexte, la population urbaine et le taux d'urbanisation de la capitale économique n'ont cessé d'augmenter avec comme corollaire la formation de nombreux quartiers spontanés dans la périphérie. Cette situation engendre d'importants problèmes d'insalubrité et de gestion urbaine (Koua, 1995).

Parmi ces problèmes, on peut citer la gestion des déchets urbains, en particulier des ordures ménagères, le balayage des rues, le curage des caniveaux, l'entretien des jardins publics et des berges lagunaires, le dégagement des trottoirs et du domaine public, sans oublier la divagation des animaux dans certains endroits. Pour remédier à cette situation, le gouvernement ivoirien avait mis en place une brigade de salubrité (janvier 1997) chargé de mener une lutte vigoureuse et coordonnée contre toutes ces nuisances en vue de restaurer la salubrité du cadre de vie des Abidjanais. L'une des actions prioritaires était d'informer et de sensibiliser la population sur la gestion de l'environnement pour une meilleure santé.

Malheureusement, on constate aujourd'hui l'absence d'un programme de sensibilisation cohérent en faveur de cette population. En réalité, les interventions épisodiques des spécialistes dans les médias ne touchent qu'une infime portion des populations concernées des quartiers précaires. Il résulte de cette situation l'indifférence et le désintérêt de ces populations vis-à-vis des actions de sensibilisation menées en vue de l'embellissement du cadre de vie. (Sissoko, 1993). L'expérience a montré que les projets communautaires de gestion des ordures conçus sans la participation de la population ont beaucoup de mal à être mis en œuvre.

\footnotetext{
${ }^{1}$ Selon une étude financée par le Fonds des Nations Unies pour la population (UNFPA) et citée par le United Nations Office for the Coordination of Humanitarian Affairs-Intégrated Regional Information Networks (IRIN), la Côte d'Ivoire comptait en 2005 1.204.966 déplacés dont 933.000 à Abidjan. Voir le site

htt://reliefweb.int/rw/rwb.nsf/vLCE/1B4ADED87C45ECE2852570C8007196 62 ?OpenDocument\&StartK ev=West+Africa\&ExpandView 
Traoré KASSOUM: De la Sensibilisation des Populations à la Gestion de l'Environnement Urbain dans les Quartiers Précaires De la Ville d'Abidjan

La sensibilisation sur les problèmes environnementaux permet à la population cible de prendre conscience des interrelations entre population, environnement et développement socio-économique tant au niveau planétaire que national et local. Elle stimule le sens de la responsabilité individuelle et collective des citoyens face à leur environnement, et contribue à conduire les individus à mieux connaître et gérer efficacement les problèmes environnementaux.

Les actions ou les programmes de sensibilisation ne présentent d'intérêt et de portée que s'ils sont prioritairement en relation concrète avec des problèmes environnementaux précis qui se posent aux populations et que celles-ci doivent résoudre. La sensibilisation en matière environnementale, objet du présent article, doit s'inscrire dans cette logique sociale, si l'on veut espérer conquérir la participation réelle des populations, dans le cadre de la préservation de l'environnement urbain. Il nous semble que c'est en mettant d'abord l'accent, à travers les actions et les programmes de sensibilisation, sur les besoins essentiels des populations (santé, habitat, éducation, travail...) que les individus pourront progressivement mieux percevoir et surtout vivre leur environnement et en avoir une conscience plus intégrée et plus globale.

Cet article examine la pertinence d'une campagne d'information et de sensibilisation de proximité en faveur des populations des quartiers précaires de la ville d'Abidjan.

Sur le plan méthodologique, l'étude combine les deux approches qualitative et quantitative. Ce travail s'appuie sur les données d'enquête et d'observation du terrain sur une période de six mois dans les quartiers précaires $\left({ }^{2}\right)$ de la ville d'Abidjan (voir la carte de la ville en annexe). L'enquête a porté sur un échantillon de 300 ménages tiré par un sondage à probabilités inégales. Les quartiers précaires enquêtés ont été choisis de façon aléatoire. Un guide d'entretien a été administré aux responsables de la gestion de l'environnement, du district et des autorités municipales.

\footnotetext{
${ }^{2}$ Ce sont Agouéto à Abobo, Sodeci-filtisac à Adjamé, Sébroko à Attécoubé, Danga bas-fond à Cocody, Zoé bruno à Koumassi, Sans fils à Marcory, Tofiato à Port-bouét, Zone industrielle à Treichville, Sicobois à Yopougon. L'enquête s'est déroulée dans le dernier semestre de 2004 et consisté à un seul passage dans les ménages.
} 
Ce travail présente dans un premier temps un aperçu de la politique de gestion de l'environnement de la ville d'Abidjan. La seconde partie porte sur la perception de l'environnement et la gestion du cadre de vie par les populations de quartiers précaires de la ville d'Abidjan. Enfin, la troisième partie analyse les conditions de travail des agents de salubrité et la crise de sensibilisation de la population.

\section{Cadre institutionnel de gestion de l'environnement urbain d'Abidjan}

La mairie centrale de la ville d'Abidjan était, à l'origine, la juxtaposition des dix (10) communes urbaines. Pour faire face aux contraintes diverses liées à la croissance de la ville, l'autorité gouvernementale a créé le district d'Abidjan qui regroupe aujourd'hui non seulement les communes mais aussi les souspréfectures $\mathrm{du}$ département d'Abidjan. Cette nouvelle entité administrative va au-delà des 10 communes d'Abidjan, car elle regroupe aussi Grand-Bassam, Dabou, Bingerville, Anyama, etc. ; par conséquent, e territoire couvert par ce district est donc très vaste. $\mathrm{Vu}$ la taille de cette unité administrative, le gouverneur du district est nommé par décret du Président de la République $(3)$. Le contrat entre le district d'Abidjan et les dix communes de la ville d'Abidjan en matière de la gestion de l'environnement reste toujours valable. Notons que le district collabore avec le Ministère de l'environnement qui est la tutelle en matière de politique environnementale. C'est elle qui développe la politique, les stratégies et les grandes actions à mener en matière environnementale.

D'après la loi n 2001-478 du 09 août 2001 portant statut du district d'Abidjan (article 6), le district d'Abidjan peut conclure toutes conventions avec l'Etat, d'autres collectivités territoriales, les établissements publics et les groupements ou les organismes privés pour mener avec eux des actions relevant de leurs compétences. Il peut aussi engager des actions complémentaires à celles de l'Etat et des collectivités territoriales de son ressort dans les domaines et les conditions fixés par la loi.

Le district d'Abidjan a pour attributions, entre autres, d'assurer :

- la protection de l'environnement,

\footnotetext{
${ }^{3}$ Article 66, titre IV, chapitre premier du journal officiel de la République de Côte d'Ivoire du 16 août 2001

156
} 
Traoré KASSOUM: De la Sensibilisation des Populations à la Gestion de l'Environnement Urbain dans les Quartiers Précaires De la Ville d'Abidjan

- la gestion des ordures et autres déchets,

- la planification de l'aménagement du territoire du district,

- la lutte contre les effets néfastes de l'urbanisation,

- la promotion et la réalisation des actions de développement économique et social,

- la lutte contre l'insécurité,

- la protection et la promotion des traditions et coutumes.

Pour promouvoir un environnement urbain sain, un certain nombre d'actions ont été mises en œuvre par la mairie. Il s'agit notamment d'un schéma général de gestion de l'environnement urbain élaboré de manière consensuelle pour gérer l'environnement des communes à Abidjan. Chaque commune devrait ainsi disposer d'un service "hygiène et environnement" dont la tâche essentielle est de lutter contre l'insalubrité de la commune avec l'appui du district d'Abidjan. Pour sa stratégie de lutte contre l'insalubrité, la commune dispose au sein du département "hygiène et environnement" de quatre services techniques spécialisés qui sont: le service hygiène, le service protection civile, le service nettoiement et le service parcs et jardins.

\subsection{Le service hygiène}

Il a pour rôle de sensibiliser la population sur la question de l'assainissement du cadre de vie. Il est composé des agents de la mairie et de certains agents des sociétés privées de sous-traitance en matière d'hygiène. Les agents doivent vulgariser auprès des habitants de la commune les principes de l'assainissement du cadre de vie, les techniques de gestion des ordures ménagères, en vue de leur assurer une meilleure santé.

Les actions de ce service s'étendent aussi à la vérification des hôtels, des toilettes publiques et des restaurants; à ceux-ci les agents de la mairie exigent une visite médicale régulière dont les résultats déterminent la fermeture ou non du restaurent ou du maquis.

\subsection{Le service de protection civile}

Le service de la protection civile dispose en général d'un cadre d'opération déterminée. Il est une extension du service d'hygiène. Il s'agit pour ce service de vérifier les conditions de travail de la population, d'exiger des travailleurs l'application des 
mesures de salubrité, pour l'assainissement du cadre de leur service, donc de leur vie. Ce service joue également un rôle de sécurité auprès des travailleurs dans la mesure où ses agents se chargent de vérifier si ces derniers sont bien équipés dans l'exercice de leur fonction, pour éviter certains accidents de travail.

Il intervient aussi dans le cadre de la gestion des ordures dans les lieux publics, dans les services et dans les usines.

\subsection{Le service nettoiement}

Ce service est chargé du curage des caniveaux à ciel ouvert, le désherbage, le balayage des quartiers. Il veille sur les lieux de rassemblement des ordures et autres déchets en attente d'enlèvement.

Ce service travaille aussi avec des sociétés telles que ASH International et Clean Bor Côte d'Ivoire, etc. Ces sociétés dans le cadre de leur intervention disposent des agents affectés au balayage des quartiers. C'est le cas de la mairie de Cocody qui a signé un contrat de collaboration avec la société clean Bor pour encadrer les femmes engagées pour accomplir cette tâche. Il en est de même pour la mairie de Port-Bouét, Marcory, Adjamé, Abobo, etc.

Le balayage et le curage des grandes artères appelées voies conventionnelles, et le ramassage des ordures relèvent de la compétence des sociétés suscitées. Il s'agit des boulevards, les voies express, les grandes rues.

Toutes ces opérations de salubrité sont faites en collaboration avec le service technique de la mairie. En effet, la collecte et l'élimination des déchets ménagers dans les dix communes d'Abidjan sont réalisées par plusieurs intervenants, dont chacun a une responsabilité précise :

- le district d'Abidjan a la responsabilité globale de la collecte et de l'élimination des ordures ménagères sur l'ensemble des dix communes ;

- les sociétés telle que ASH International, Clean Bor, etc., sont des sociétés privées qui ont obtenu un contrat d'enlèvement des ordures avec le district d'Abidjan; chacune d'elle s'occupe d'une subdivision de la ville ; 
Traoré KASSOUM: De la Sensibilisation des Populations à la Gestion de l'Environnement Urbain dans les Quartiers Précaires De la Ville d'Abidjan

- la brigade de salubrité du Ministère de l'Environnement et du Cadre de vie a la responsabilité de concevoir le programme national de d'élimination des déchets urbains.

Malgré cet arsenal de mesures, le taux d'enlèvement des ordures n'atteint pas les 100\%. Par conséquent, ce sont les communes elles-mêmes qui s'occupent de l'enlèvement des déchets non pris en compte dans le contrat, notamment les branchages, les déchets inaccessibles aux camions des sociétés.

Ainsi, les sociétés qui ont obtenu le contrat d'enlèvement avec le district d'Abidjan enlèvent-elles environ $80 \%$ des déchets ménagers. Les $20 \%$ restant sont enlevés par les communes, généralement équipées de petits engins de collecte.

Traditionnellement, la chaîne de collecte et d'élimination des ordures par les sociétés comprend :

- le stockage à domicile dans les récipients à usage individuel agrées par des sociétés telle que ASH (les poubelles Mipa, les demi-fûts de 75 à 100 litres) ou stockage dans les poubelles, coffres ou amplirolles à usage collectifs. $\left({ }^{4}\right)$,

- la prise en charge des déchets des poubelles individuelles ou récipients collectifs par les camions des sociétés de ramassage d'ordure,

- l'acheminement des déchets vers la station de transfert de Williamsville (pesage et stockage des déchets),

- le transport des déchets par les camions gros porteurs vers la décharge d'Akouédo et élimination par étalage des déchets.

Pour ce qui est du coût de la collecte et de l'élimination des déchets, estimation s'élevait à 8 milliards de Frs CFA pour l'année 1997-1998 en raison de la production journalière de 2.000 à 2.300 tonnes d'ordures. Toutes les communes contribuent financièrement à hauteurs de $40 \%$ de leur budget à ces opérations pour la salubrité des communes (Direction de l'Environnement, 2001).

Outre ces opérations pratiques de salubrité, la société ASH International va recommander aux populations, en collaboration

\footnotetext{
${ }^{4}$ N'goran kouassi (Cyraque) : Etude préliminaire sur la gestion des déchets ménagers, Abidjan, Ministère du Logement, du cadre de vie et de l'Environnement, Octobre 1990, P8.
} 
avec les communes, l'utilisation des sacs appelés " $\mathrm{H}$ " pour une meilleure gestion des ordures.

\subsection{Le service parcs et jardins}

Ce service a pour rôle l'entretien des espaces verts, la maintenance de la salubrité, l'étayage des arbres, l'entretien de la berge lagunaire, l'entretien des jardins publics.

Certaines communes n'ont pas concrètement entrepris des actions pour lutter contre la pollution de la berge lagunaire. En principe le service parcs et jardins intervient pour la gestion des ordures ménagères versées en bordure de la lagune et dans les jardins publics par les riverains. Toutefois, il est à signaler que depuis 2004 la mairie de Plateau a amorcé la réhabilitation de la baie lagunaire du Plateau en créant des espaces de jeux, de repos, etc.

Certaines mairies ne disposent pas d'un tel service pour les raisons inhérentes à la gestion de leur structure. Elles confient cet aspect à des sociétés privées telles que Clean Bor Côte d'Ivoire, Arôme, Lassire-déchet, etc. C'est le cas de la mairie d'Adjamé, d'Abobo, de Yopougon, Port-Bouët.

En conclusion de ce premier point, on peut dire que le cadre institutionnel de gestion de l'environnement urbain est relativement développé. En effet, il existe plusieurs structures intervenant dans les actions de promotion de la salubrité urbaine. Toutefois les résultats du travail accompli sur le terrain par ces structures sont loin d'atteindre les objectifs poursuivis par la politique de préservation de l'environnement urbain. Les perceptions des habitants des quartiers périphériques le montrent ; c'est l'objet du point suivant.

\section{Perception de l'environnement et gestion du cadre de vie par les populations de quartiers précaires de la ville d'Abidjan.}

La perception de l'environnement également désignée sous le vocable de conscience écologique est l'ensemble des représentations et significations qu'un individu ou une collectivité se fait de son environnement physique et qui en retour déterminent ses comportements et ses attitudes vis-à-vis de l'environnement (Koffi, 1996). Cette partie de l'étude a pour objet d'analyser les opinions des populations des quartiers précaires ont 
Traoré KASSOUM: De la Sensibilisation des Populations à la Gestion de l'Environnement Urbain dans les Quartiers Précaires De la Ville d'Abidjan

de l'environnement et l'attention qu'elles prêtent à l'hygiène de leur cadre de vie.

\subsection{La perception des ménages des quartiers précaires relative à l'environnement}

La compréhension de l'environnement peut être liée à l'appartenance à un groupe (dimension culturelle) et au niveau $\mathrm{d}$ 'instruction de l'individu. Pour mieux cerner les problèmes d'insalubrité qui se posent dans les quartiers précaires de la ville d'Abidjan, il a été nécessaire de connaître ce que savent les populations de l'environnement. Le graphique ci-dessous retrace les réponses que donnent les enquêtés des quartiers précaires. Ici, les modalités étant indépendantes les unes des autres, un chef de ménage à la possibilité de cocher plusieurs réponses.

Graphique 1 : Perception de l'environnement par les ménages

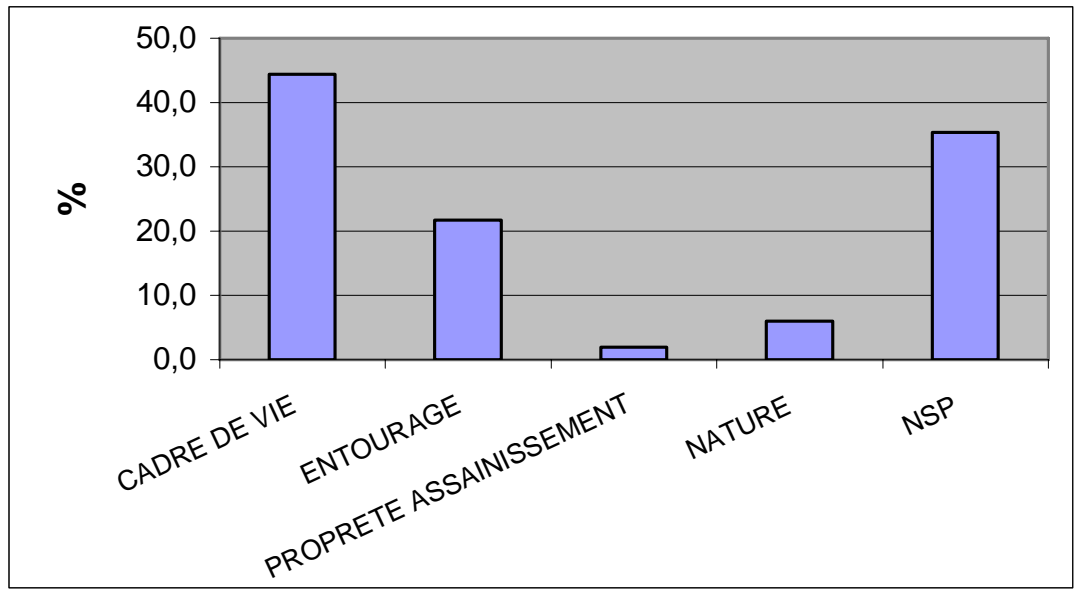

L'enquête a révélé que la grande majorité des ménages interrogés (44\%) assimilent l'environnement au cadre de vie, tandis qu'une autre portion relativement importante $(21 \%)$ l'assimile à leur entourage immédiat. Par contre, plus d'un tiers (35\%) des enquêtés n'appréhende pas l'environnement. Ce résultat traduit en définitive une perception peu diversifiée de l'environnement par les Abidjanais.

Concernant les sources d'information sur l'environnement, l'audio-visuel joue un rôle prépondérant, avec 50 des personnes 
interrogées renseignées par ce canal. Celui-ci est suivi de loin par les mairies et les amis. Les autres sources occupent une place négligeable. En d'autres termes, la radio et la télé occupent une place stratégique dans la communication en matière d'environnement. Il est cependant difficile d'évaluer le niveau réel d'information, voire de sensibilisation de la population concernée, en l'absence de données sur le contenu des informations diffusées et les changements de comportements observés. Ce type de données devra être collecté dans une étude ultérieure.

Graphique 2 : Source d'information sur la question de l'environnement

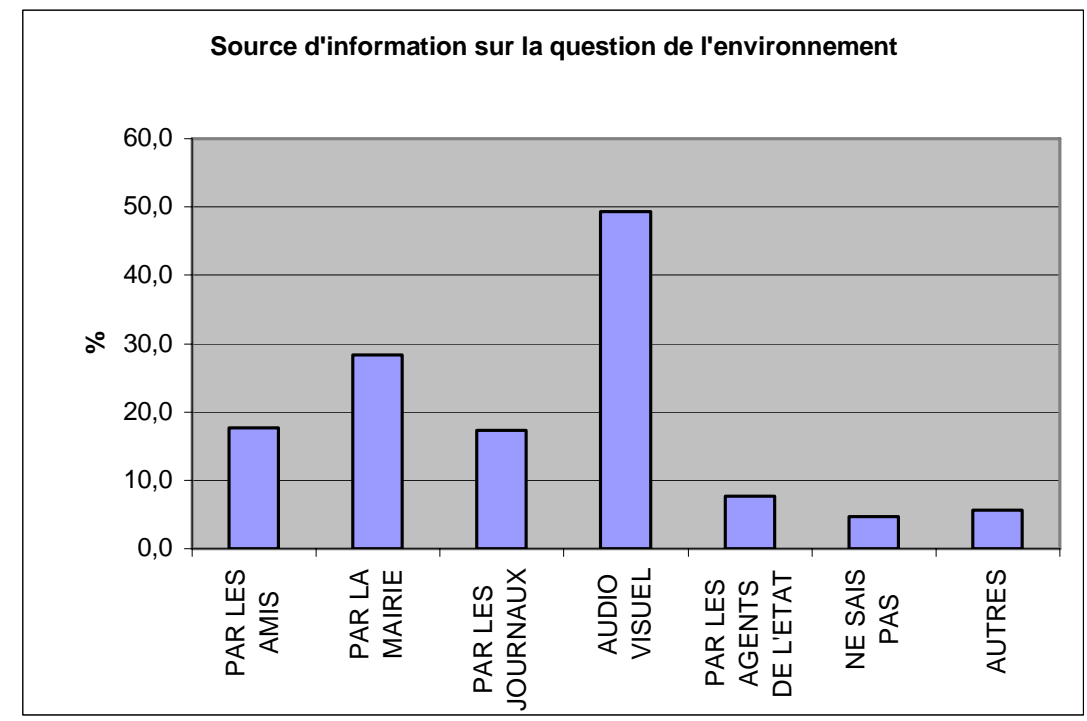

\subsection{La pratique de l'hygiène dans les quartiers précaires}

L'enquête a saisi également la pratique des règles d'hygiène dans les ménages enquêtés, en cherchant notamment à savoir comment ces populations gèrent leur cadre de vie. A cet effet, les questions ont été posées sur :

- le mode d'évacuation des ordures (avez-vous des poubelles ?) ;

- le mode d'évacuation des eaux usées (où déversez-vous les ordures ? existe-t-il des caniveaux ?) ;

- l'opinion sur les actions des mairies et du gouvernement, 
Traoré KASSOUM: De la Sensibilisation des Populations à la Gestion de l'Environnement Urbain dans les Quartiers Précaires De la Ville d'Abidjan

\subsubsection{Le mode d'évacuation des ordures ménagères}

L'enquête a révélé que l'usage des poubelles individuelles par les ménages est assez courant puisque plus de trois quarts des ménages $(78 \%)$ en possèdent. La qualité des poubelles utilisées influent cependant sur le stockage et l'évacuation des déchets. Ainsi, il apparaît que le stockage des ordures dans les ménages se fait essentiellement dans des récipients solides (95\%). Toutefois, une frange des ménages (4\%) utilise des sacs en plastique, tandis que les autres $(1 \%)$ déversent directement leurs ordures dans la nature.

Les ménages qui ont déclaré ne pas disposer de poubelle chez eux, jettent directement les ordures dans la nature. Pour $23,9 \%$ des chefs de ménage, la lagune est utilisée, $6 \%$ jettent dans la brousse, $16,4 \%$ jettent près de la maison et $17,9 \%$ dans autres lieux qui sont en général des trous, caniveaux, au bord des routes, dans la lagune. Ce mode d'évacuation des ordures ménagères est à l'origine de la pollution solide, sources de nuisances diverses et de risques sanitaires. En revanche, 35,8\% préfèrent mettre à la poubelle publique qui après sont enlevées par les véhicules de ramassage des ordures.

Les poubelles rencontrées dans ces quartiers sont généralement sans couverture. Or c'est à proximité de ces poubelles que se déroulent la majorité des activités commerciales $\mathrm{du}$ secteur informel, notamment les restaurants, les bars de Tchapalo, de vin de palme et autres activités destinées à la consommation. Il en résulte des risques élevés de contamination et de maladies infectieuses et parasitaires.

\subsubsection{Le mode d'évacuation des eaux usées}

Le réseau de drainage des eaux usées est quasi inexistant dans les quartiers étudiés. En effet, 98\% des ménages rejettent leurs eaux usées dans la nature, provoquant ainsi le multiplication des flaques d'eaux stagnantes propices au développement des larves de moustiques, ce qui concourt au risque paludéen dans la ville d'Abidjan. 
Graphique 3 : Représentation des lieux d'évacuation des eaux usées

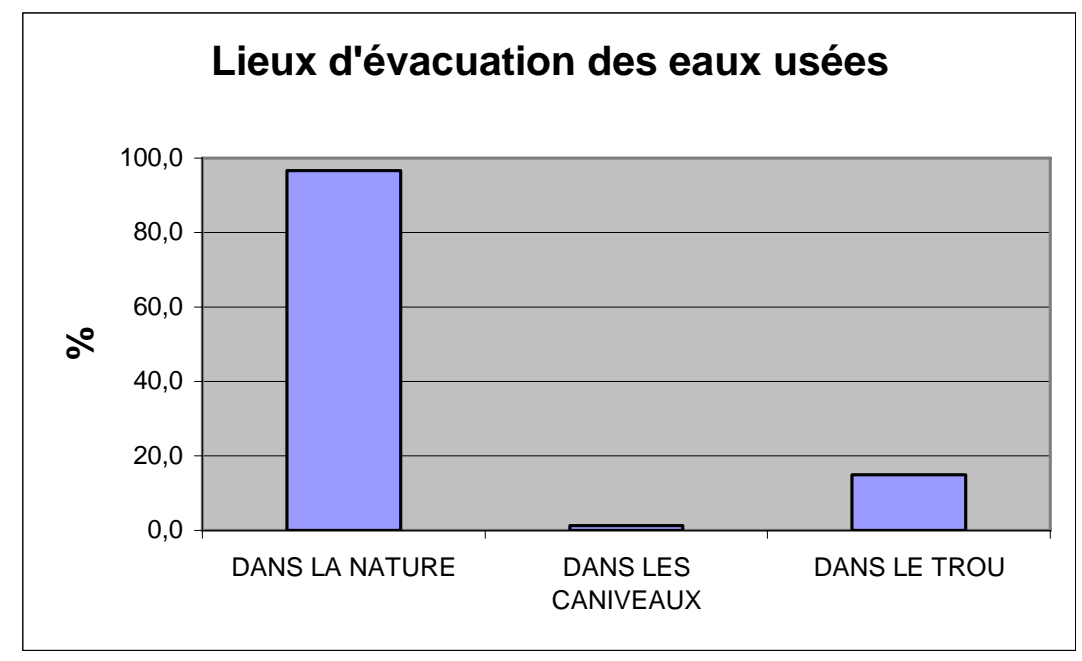

Le traitement des eaux usées dans les quartiers précaires n'est guère satisfaisant. En effet, la majorité des ménages évacuent leurs eaux usées dans la nature (soit à proximité de leur habitation, soit dans la lagune). A ceux là, il faut ajouter les ménages qui utilisent les trous ou les fosses sceptiques. Dès lors, on peut dire que les eaux usées ne sont pas évacuées correctement. Les ménages qui utilisent les caniveaux pour l'évacuation de leurs eaux usées sont minoritaires (4\%). Ce sont les ménages des fonctionnaires et des agents du privé qui habitent dans les zones d'extension proches des quartiers résidentiels.

Les données montrent qu'une proportion relativement importante des populations des quartiers précaires a des comportements dommageables à l'environnement. La non utilisation des lieux conventionnels de dépôt des ordures ménagères entraîne l'insalubrité du cadre de vie des populations des quartiers précaires.

Par ailleurs, malgré les efforts consentis par les pouvoirs publics en installant des poubelles publiques et des coffres à ordures dans les quartiers ainsi que l'utilisation des sacs " $\mathrm{H}$ " par l'entremise des responsables communaux pour une meilleure gestion du cadre de vie, la population n'évacue pas toujours les ordures de manière appropriée, c'est-à-dire aux endroits indiqués. Les ordures bouchent les caniveaux surtout au niveau des marchés et les bordures des routes, engendrant ainsi de sérieux problèmes d'environnement. 
Traoré KASSOUM: De la Sensibilisation des Populations à la Gestion de l'Environnement Urbain dans les Quartiers Précaires De la Ville d'Abidjan

L'enlèvement des poubelles est surtout l'affaire des enfants et des femmes de ménages. En effet, la majorité des personnes enquêtées exercent des activités libérales (à ceux là, il faut ajouter quelques fonctionnaires et les agents du privé) et sont presque toujours absents du domicile. Les ménagères qui sont quelques fois présentes confient cette tâche aux enfants car elles sont occupées à d'autres activités de ménage ou de petits commerces.

Le problème qui se pose ici est surtout de savoir si les enfants et les servantes de maison à qui revient la charge de vider la poubelle, reçoivent les consignes nécessaires de la part de leurs parents ou leurs employeurs pour déverser les ordures au bon endroit. Cela ne semble pas être le cas car dans tous les quartiers sillonnés lors de notre enquête, les ordures sont parfois déversées à même le sol à proximité des logements, et aux abords des routes; tandis que les coffres destinés à les recevoir sont presque vides. Le manque d'éducation et la négligence sont à l'origine de cette situation susceptible de provoquer la dégradation de du cadre de vie.

\subsubsection{Les opinions des enquêtés sur les actions des mairies}

Les opinions de la population sont en général très mitigées : en effet plus de la moitié de la population interrogée (58\%) juge l'action des pouvoirs publics insuffisante, tandis qu'un peu plus d'un quart $(27 \%)$ la trouve acceptable. Une frange de personnes interrogées (15\%) est sans opinion. En définitive, ce résultat traduit une certaine insatisfaction de la population des quartiers concernés devant les interventions des pouvoirs publics.

Pour connaître les réels motifs des réactions des populations sur les relations qui existent entre elles et la mairie, nous avons cherché à savoir si la mairie les convie aux réunions concernant la gestion de leur environnement. Si elle est sensible aux problèmes qui se posent à leur cadre de vie. Les réponses obtenues se présentent comme suit dans le graphique suivant: 
Graphique 4: Opinions des enquêtés sur la relation de la mairie/populations

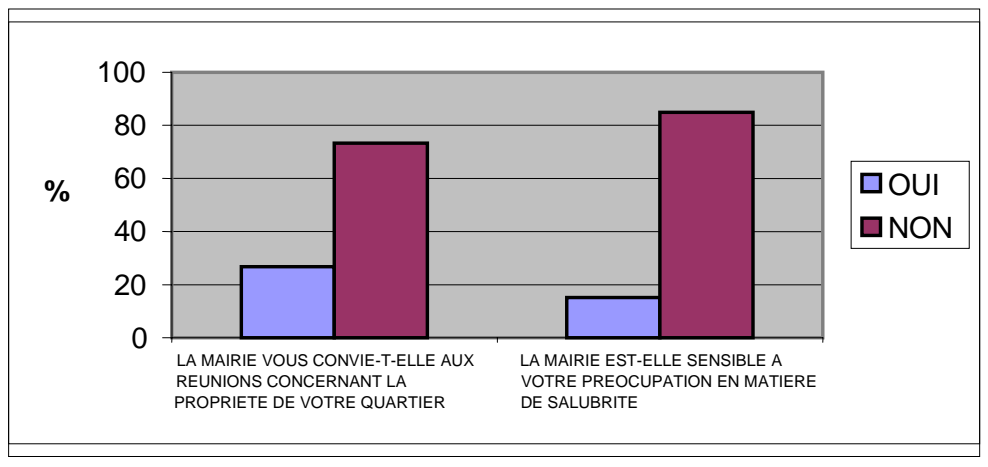

De l'avis des enquêtés, respectivement 26,7\% (soit 80 personnes) et $15,1 \%$ (soit 45 personnes), la mairie les convie aux réunions et elle est sensible à leur problème de salubrité. Pour preuve, ces derniers se réfèrent aux réunions que les mairies initient souvent avec les chefs des quartiers. La présence des agents d'hygiène qui sillonnent la commune pour l'entretien des artères et des rues justifie aussi cette réaction. Mais en réalité, le constat sur le terrain montre que les résultats escomptés de toutes ces actions menées ne sont pas probants. C'est ce qui fait dire respectivement à $73,3 \%$ (soit 220 personnes) et $84,9 \%$ (soit 254 personnes) que la mairie ne les convie pas aux réunions et n'est pas sensible à la dégradation de leur cadre de vie. Pour ces derniers, la mairie ne travaille pas bien pour la salubrité des quartiers.

En effet, la crise du ramassage des ordures observée à certains moments dans la commune, est perçue aussi comme la preuve de l'inefficacité des mairies. Ce reproche fait aux mairies trouve son explication dans leur rattachement au district d'Abidjan pour la collecte et l'élimination des déchets dans les communes. Malgré ce fait, il y a lieu de remarquer qu'aux yeux des administrés, l'autorité communale reste la première responsable des problèmes de la commune et doit donc résoudre celui de l'insalubrité. Toutefois certaines Mairies ne peuvent pas totalement faire face à cette tâche parce que leurs recettes seraient insuffisantes. Le budget ne permet pas de faire de grands travaux de réhabilitation. 
Traoré KASSOUM: De la Sensibilisation des Populations à la Gestion de l'Environnement Urbain dans les Quartiers Précaires De la Ville d'Abidjan

\subsubsection{Les opinions des enquêtés sur les actions du gouvernement}

L'avis des enquêtés sur les actions menées par le gouvernement s'est limité à la connaissance qu'ils ont des campagnes de sensibilisation entreprises par les pouvoirs publics. Les données recueillies révèlent que dans leur immense majorité (79\%), les populations ne sont pas au courant de ces initiatives. Ceci compromet évidemment leur adhésion aux actions menées.

Graphique 5 : l'avis des enquêtés sur la campagne de sensibilisation du gouvernement

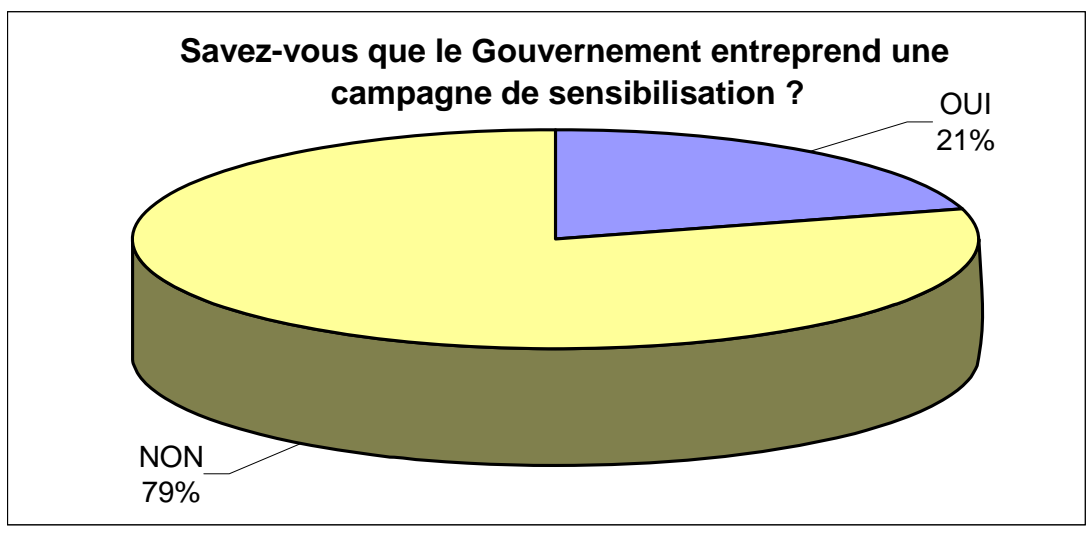

Ce résultat montre que face aux difficultés économiques, les populations accordent moins d'intérêt aux questions environnementales. Elles sont en effet plus préoccupées par la lutte pour la survie donc par les questions économiques. Ce manque d'intérêt à l'information environnementale contribue à accentuer les atteintes à l'environnement avec les risques sanitaires qui en résultent. Le faible niveau d'instruction des populations concernées justifie en partie ce manque d'intérêt à l'information parce qu'elles ne comprennent pas la portée du message diffusé surtout dans la langue française. Ce qui signifie que le canal d'information mis en place n'est pas adapté aux réalités du terrain. Il apparaît en effet que plus de la moitié des enquêtés $(53,2 \%)$ avoue ne pas saisir le message véhiculé dans la campagne de sensibilisation. Ceci signifie que la campagne de sensibilisation $\mathrm{n}^{\prime}$ atteint pas son objectif qui est non seulement d'informer mais aussi de provoquer un changement de comportement. Dans ces conditions, on peut s'interroger sur l'impact réel la rentabilité sociale de ces campagnes de sensibilisation. 
Les changements opérés à la suite des recommandations de la campagne de sensibilisation surtout sur l'entretien du cadre de vie s'observent chez $37,9 \%$ des enquêtés (qui utilisent des produits chimiques), $44,8 \%$ des enquêtés gèrent bien des déchets en les regroupant dans un lieu approprié pour éviter les désagréments. $55,2 \%$ des enquêtés disent faire des efforts pour rendre propre leur cadre de vie. Par contre, $45,5 \%$ des enquêtés soutiennent que rien n'a changé dans leur environnement contre 39,4\% qui affirment ne pas comprendre le message véhiculé. La vie en groupe est difficile pour $24,2 \%$ des enquêtés. Pour ces derniers, le comportement de certaines personnes représente des freins pour la gestion de l'environnement.

En somme, la gestion de l'environnement en général et celle des ordures ménagères en particulier sont un problème sérieux à suivre de près. Malgré le renforcement des moyens, l'on constate que le comportement de certaines personnes restera toujours un obstacle majeur parce que celles-ci ne facilitent pas la tâche aux structures de gestion. Cependant, les autorités ont leur part de responsabilité dans le dysfonctionnement de la gestion. Les entreprises adjudicataires accusent souvent les autorités de gestion du retard prolongé de paiement des prestations de service. Ce qui provoque souvent une crise entre ces sociétés et les autorités. L'exemple du conflit éclaté entre la société Clean Bor Côte d'Ivoire et du District d'Abidjan en 2003 en est une preuve. Les zones confiées à cette société souffrent de pollution, du fait d'une collecte des ordures ménagères inappropriée.

On peut dire, pour conclure ce point, que la gestion des déchets urbains souffre de dysfonctionnements préjudiciable à la préservation de l'environnement urbain et du cadre de vie de populations des quartiers périphériques. Les conditions techniques de travail sont aussi responsables de cette situation.

\section{Les conditions techniques de travail des agents de salubrité et la crise de sensibilisation de la population}

\subsection{Les conditions techniques de travail des agents de salubrité}

Les données recueillies montrent que les conditions techniques dans lesquelles le travail s'effectue sont inappropriées. Malgré la restauration des services techniques des mairies, les moyens ne sont pas adaptés aux objectifs de la lutte contre l'insalubrité définis par l'autorité communale. Il n'y a pas assez de 
Traoré KASSOUM: De la Sensibilisation des Populations à la Gestion de l'Environnement Urbain dans les Quartiers Précaires De la Ville d'Abidjan

camions pour le ramassage des ordures inaccessibles aux camions. Le matériel pour le désherbage et l'étayage des arbres reste artisanal. La desserte des quartiers par les agents municipaux se fait à pied. La rémunération reste insignifiante par rapport aux efforts exigés. Tout cela constitue autant de problèmes qui pour les agents de salubrité entrave la bonne marche de leurs différentes actions. La population enquêtée (malgré les problèmes d'insalubrité qu'elle vit) reconnaît que les agents travaillent dans des conditions techniques difficiles. Pour elle, cela s'explique par le fait que les mairies ne font pas des efforts suffisants pour améliorer les conditions techniques dans lesquelles travaillent les agents de salubrité.

En ce qui concerne les résultats, les agents de salubrité se plaignent d'être constamment confrontés à la réticence de la population, quant à l'application des mesures de salubrité qu'ils leur enseignent pour l'assainissement de leur cadre de vie. Selon ces derniers, seulement une minorité des ménages prennent en considération ces mesures. Ce qui entraîne la dégradation du cadre de vie des familles.

Cette attitude de la population s'explique par le fait que les agents de salubrité ne disposent que d'un léger pouvoir de répression, en cas de refus d'application des mesures de salubrité. Ainsi, ces derniers réclament-ils un renforcement de leurs pouvoirs, avec l'adoption de certaines lois visant à punir ceux qui portent atteinte à l'environnement. Les textes réglementaires en matière d'hygiène sont insuffisants. Ce qui a pour conséquence les comportements anarchiques des producteurs de déchets et de certains éliminateurs.

Notons également que la crise économique qui touche les populations abidjanaises notamment celles des quartiers précaires ne leur permet pas d'accorder suffisamment d'attention à la préservation de leur cadre de vie. En effet, il est difficile, quand on est pauvre, de ne pas porter atteinte à l'environnement et au cadre de vie. On n'a peut-être pas besoin d'être riche pour être propre. Cependant, dans les zones urbaines défavorisées, la pauvreté se conjugue avec la dégradation du cadre de vie.

Entassés dans des maisons étroites, certaines familles ont du mal à assurer la salubrité de leur cadre de vie, faute d'espace. Certaines d'entre elles sont financièrement incapables de se procurer de l'eau tous les jours pour les besoins d'hygiène 
corporelle et celle du milieu. Certaines familles arrivent à le faire une et deux fois par semaine, en se limitant au simple balayage. Cet état de fait ne permet pas d'obtenir un résultat probant. On a pu observer dans certains endroits une amélioration de la salubrité des cours communes grâce aux actions de ces agents.

Apprendre à la population à assurer la salubrité de leur cadre de vie est une action noble, mais qui exige un minimum de connaissances voire d'éducation, de motivation individuelle et de moyens matériels et financiers au niveau communautaire. De ce fait, ils sont déterminés à continuer leurs actions, si les conditions techniques leurs sont favorables. Les difficultés relevées sont à l'origine de la crise de sensibilisation observée.

3.2 La crise de sensibilisation de la population: réticence de la population face aux mesures de salubrité

Les agents de salubrité des mairies et ceux du district d'Abidjan sont confrontés tous les jours à la réticence de la population qui refuse d'appliquer convenablement les mesures de salubrité qu'on leur donne ou du moins qu'on leur impose. En réalité, cette attitude de la population des quartiers précaires face aux agents de salubrité, est consécutive à une crise de sensibilisation de cette population pour l'assainissement de leur cadre de vie. Autrement dit, malgré les efforts consentis par les autorités municipales et celles du district d'Abidjan, la sensibilisation de la population des quartiers précaires de la ville d'Abidjan pour l'hygiène du cadre de vie n'a pas encore eu les résultats escomptés.

Ainsi, assiste-t-on régulièrement à des bagarres entre agents de salubrité et ménagères. En effet, les fréquents reproches faits par des agents de salubrité à l'endroit des habitants de certaines cours pour des raisons d'insalubrité, est la preuve que le message passe difficilement. En revanche, les populations estiment que les autorités ne font pas le travail de collecte des ordures comme il se doit alors qu'elles font des efforts de rassembler les ordures dans les endroits indiqués.

Les ordures continuent d'être jetées dans les rues, les jardins publics et les berges de la lagune. Tout ceci atteste bien que l'impact de la sensibilisation de la population dans les communes d'Abidjan, en particulier les quartiers précaires, est encore faible. 
Traoré KASSOUM: De la Sensibilisation des Populations à la Gestion de l'Environnement Urbain dans les Quartiers Précaires De la Ville d'Abidjan

Le fait social qui se dégage finalement des données recueillies est que les populations des quartiers précaires, analphabètes dans leur immense majorité, considèrent généralement que les problèmes environnementaux, en particulier la gestion des déchets, est l'affaire des seuls pouvoirs publics. En réalité elles ont du mal à comprendre que l'implication des ménages et des individus dans les programmes de préservation de l'environnement est déterminante pour le succès de ces programmes. Ceci est particulièrement valable pour les programmes mis en œuvre par les mairies.

$\mathrm{Du}$ fait de leur analphabétisme, ces populations ne parviennent pas à comprendre les liens existant entre les problèmes environnementaux immédiats (ordures ménagères, assainissement, insalubrité...) et la santé humaine.

\section{CONCLUSION}

Il se dégage de l'analyse des données que la sensibilisation sur les problèmes environnementaux en milieu urbain notamment dans les quartiers précaires reste insuffisante. Il résulte de ce déficit de sensibilisation la faible mobilisation des populations concernées pour la résolution des problèmes rencontrées.

La prise de conscience de l'interaction entre les activités humaines, l'environnement et la santé reste un objectif à atteindre. Il faut donc continuer à sensibiliser le public aux problèmes d'environnement et le faire participer davantage à la recherche des solutions. C'est cela qui pourrait renforcer chez chaque individu le sens de la responsabilité personnelle à l'égard de l'environnement et la volonté de s'engager en faveur d'une gestion rationnelle.

C'est pourquoi les opérations de sensibilisation auprès des populations devront prendre en compte toutes les catégories sociales tout en mettant un accent sur les quartiers précaires où les problèmes d'environnement notamment d'insalubrité se posent avec beaucoup d'acuité. L'environnement que l'on veut préserver ou améliorer ne peut être l'affaire des seuls pouvoirs publics. La participation de tous les segments de la société est indispensable pour atteindre cet objectif. La mise en place de comités locaux d'action environnementale avec l'appui des autorités administratives et municipales, des ONG et des entreprises industrielles et commerciales pourrait y contribuer. L'objectif 
d'une telle initiative est de promouvoir l'approche participative pour la résolution des problèmes d'environnement urbain.

\section{BIBLIOGRAPHIE}

ATTAHI (K.), (1993) : Evaluation des reformes de gestion urbaine: villes africaines en crise, gérer la croissance urbaine au sud du Sahara, in Stren R. et White R. Paris, l'harmattan, 341p.

BAD, (1996): Sensibilisation des Directeurs centraux, régionaux, départementaux de l'éducation sur le programme de l'éducation environnementale scolaire en Côte d'Ivoire, le cahier $n^{\circ} 3 \mathrm{du}$ participant, 58p

CREPA, (2002), Assainissement écologique: plaidoyer pour la lutte contre la pauvreté, in info CREPA, avril-juin, pp 4-10.

DCGTx, (1995): Amélioration des services urbains dans les quartiers sous équipés de l'agglomération d'Abidjan: les caractéristiques des quartiers et proposition d'action prioritaires, Tome II, Abidjan, AUA, 167p.

DOKOMON (B.), (1992): L'assainissement en Côte d'Ivoire "les problèmes liés à la gestion de l'assainissement et stratégie d'actions", mémoire de DEA, université de Cocody, IES, Abidjan, $59 \mathrm{p}$.

GIDIS, (2005) : Le journal des sciences sociales, revue scientifique $\mathrm{du}$ groupement interdisciplinaire en sciences sociales, $\mathrm{n}^{\circ} 2$ décembre 2005.

WYSS (K.), YEMADJI (N'.), CISSE (G.) TANNER (M.). (2001) : Gestion par leur occupants d'environnement urbains défavorisés au sahel in Sempervira, institut Tropical Suisse, revue ${ }^{\circ} 10,142 p$.

KOFFI (k.) (1996): Les obstacles sociologiques à la préservation de l'environnement en milieu urbain africain, mémoire de DEA, Université de Cocody, IES, Abidjan, 64p.

KOUA LEON K., (1995): Document de séminaire de formation des agents des médias aux concepts et aux problèmes environnementaux, 9p. 
Traoré KASSOUM: De la Sensibilisation des Populations à la Gestion de l'Environnement Urbain dans les Quartiers Précaires De la Ville d'Abidjan

MAïGA (M.), (1998) : La problématique de la lutte contre l'insalubrité à Abidjan: le cas de la commune de Treichville, mémoire de maîtrise, Université de Cocody, IES, Abidjan, 86p.

MINISTERE DE L'ENVIRONNEMENT ET DU CADRE DE VIE (2001) Direction de l'environnement: Stratégie et Programme National de gestion durable des déchets solides ; RCI, 57p.

SISSOKO A., (1993): Rapport sur les domaines et actions prioritaires en matière d'éducation environnementale: aspects informations et sensibilisation, PNAE-CI, Abidjan, 46p.

YAPI DIAHOU (A.), (2000): Baraques et pouvoirs dans l'agglomération abidjanaise, Paris, l'harmattan, 456p. 